Research Paper

\title{
Targeting BRD4 prevents acute gouty arthritis by regulating pyroptosis
}

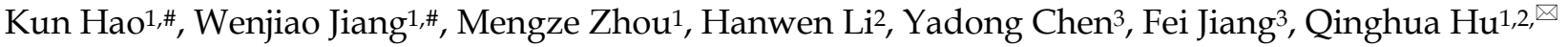 \\ 1. State Key Laboratory of Natural Medicines, Key Laboratory of Drug Metabolism and Pharmacokinetics, China Pharmaceutical University, Nanjing 210009, \\ PR China \\ 2. School of Pharmacy, China Pharmaceutical University, Nanjing 211198, PR China \\ 3. School of Science, China Pharmaceutical University, Nanjing 211198, PR China \\ \# These authors contributed equally to this study \\ $\bowtie$ Corresponding author: Associate Professor Qinghua Hu, State Key Laboratory of Natural Medicines, China Pharmaceutical University, Nanjing 210009, PR \\ China. Email: huqh@cpu.edu.cn \\ (C) The author(s). This is an open access article distributed under the terms of the Creative Commons Attribution License (https://creativecommons.org/licenses/by/4.0/). \\ See http://ivyspring.com/terms for full terms and conditions.
}

Received: 2020.03.19; Accepted: 2020.10.04; Published: 2020.10.17

\begin{abstract}
Background: Acute gouty arthritis is a common inflammatory arthropathy resulting from urate deposition in joints during persistent hyperuricemia. Nevertheless, effective therapeutic strategies are still unavailable. Here, we propose the crucial role of bromodomain-containing protein 4 (BRD4) in acute gouty arthritis.

Methods: Therapeutic effect of BRD4 specific inhibitor JQ-1 on acute gouty arthritis was evaluated in vivo and in vitro. Pyroptosis was analyzed by Caspase-1/PI double staining and cleavage of gasdermin D (GSDMD). Expression of key factors involved in BRD4/NF-KB/NLRP3/GSDMD signaling pathway were measured by western blot, and colocalization of NLRP3 and ASC was detected using immunofluorescence. In addition, the role of BRD4 on monosodium uric acid crystals (MSU)-induced pyroptosis was verified in BRD4 siRNA-transfected THP-1 cells.

Results: Pretreatment of JQ1 and BRD4 siRNA significantly suppressed pyroptosis and inhibited activation of p65 NF-KB signaling as well as NLRP3 inflammasome in THP-1 cells exposed to MSU. In vivo, JQ-1 administration could effectively attenuate joint swelling and synovial inflammation in rats treated by intra-articular injection of MSU. More importantly, MSU led to macrophage pyroptosis and Brd4/NF-KB/NLRP3/GSDMD signaling induction in rat synoviums, which was improved by JQ-1.

Conclusions: Our study identifies the role of BRD4 in MSU-induced pyroptosis through regulating NF-KB/NLRP3/GSDMD signaling pathways, which provides a potential target for treatment of acute gouty arthritis.
\end{abstract}

Key words: BRD4; Acute gouty arthritis; Pyroptosis; NLRP3 inflammasome

\section{Introduction}

Gout is regarded as a common inflammatory arthropathy characterized by hyperuricemia and accumulation of monosodium urate crystals (MSU) in joints [1]. Deposition of MSU caused by the excessive serum uric acid levels could trigger an intense inflammatory process with pain along with tophi, leading to exaggeration of gouty inflammation [2]. MSU crystallization-induced gout can occur in joints, peri-articular tissues and kidneys [3]. Among them, joints are frequently subjected to gouty attacks, eventually resulting in acute gouty arthritis. Thus, articular injection of MSU is usually applied to establish the animal models to mimic acute gouty arthritis as previous studies shown [4].

At present, it has been reported that MSU administration leads to excessive activation of NLRP3 inflammasome in pathologies [5]. Assembly of NLRP3 inflammasome depends on combination of Nod-Like Receptor Protein 3 (NLRP3), and apoptosis associated speck like protein (ASC), as well as cleavage of cysteinyl aspartate specific proteinase-1 precursor (pro-Caspase-1), which functions as pattern recognition receptors in response to stimuli [6]. The secretion process of inflammation cytokines IL- $1 \beta$ is 
mediated by the NLRP3 inflammasome activation governing the following activation of Caspase- 1 to promote the maturation of pro-IL-1 $\beta[7,8]$. Notably, Caspase- 1 activation is found to break the linker between the amino-terminal gasdermin- $\mathrm{N}$ and carboxy-terminal gasdermin-C domains in gasdermin D (GSDMD), leading to formation of membrane pores, which has been regared as a key step of pyroptosis [9-11]. Pyroptosis processing could be observed, in which cell edema, secretion of pro-inflammatory intracellular events and pore formation in the cell membrane once pyroptosis started [12].

As a representative member of the BET (Bromodomain and Extra-Terminal) protein family, the bromodomain-containing protein 4 (BRD4) has been reported to mediate regulation of NF- $\mathrm{KB}$ signaling via acetylated RELA [13]. Current studies indicated that small-molecule BRD4 inhibitor JQ-1 ameliorated rheumatoid arthritis via blocking NF-kB activation [14, 15]. However, it is still unclear about the mechanism behind the protective effect of JQ-1 on regulating synovial inflammation. Here, we uncovered a role for bromodomain-containing protein 4 (BRD4) in acute gouty arthritis and explored how it is involved in the pathogenesis of MSU-induced acute gouty arthritis models.

\section{Methods and materials}

\section{Reagents}

JQ-1 and uric acid were purchased from Sigma-Aldrich (St. Louis, USA). Bicinchoninic acid Protein Quantification Kit was supplied by Vazyme Biotech (Nanjing, China). Interleukin-1 $\beta$ (IL-1 $\beta$ ) enzyme-linked immunosorbent assay kit was provided by R\&D Systems (Minneapolis, MN, USA). Primary antibodies were listed in Tables. Secondary antibodies conjugated with Alexa Fluor ${ }^{\circledR} 488$, Alexa Fluor ${ }^{\circledR}$ 568, and Alexa Fluor ${ }^{\circledR} 647$ were purchased from Abcam (Cambridge, UK). FLICA® 660 in vitro Caspase-1 Detection Kit was purchased from ImmunoChemistry Technologies (Bloomington, USA).

\section{Animals}

30 Male Adult Sprague-Dawley rats (250-280g) were obtained from Shanghai SIPPR-BK Lab Animal Co. Ltd. and were allowed to acclimate to the laboratory conditions for one week prior to experiments. All animal studies were performed according to the Guide for the Care and Use of Laboratory Animals and approved by the China Pharmaceutical University Animal Ethics Committee.

\section{Preparation of MSU crystal}

MSU crystal was prepared as our previous study [16]. The uric acid solution was dissolved in double-distilled water at room temperature, followed by crystal formation at $4^{\circ} \mathrm{C}$ overnight. After filtration, the precipitate was then dried at $70^{\circ} \mathrm{C}$ for $4 \mathrm{~h}$, grounded into a fine powder, and sterilized by heating at $180^{\circ} \mathrm{C}$ for $2 \mathrm{~h}$. The MSU crystals were suspended using sterile PBS for administration.

\section{Animal models and treatment regimes}

To investigate the anti-inflammatory activity of JQ-1 in vivo, we establish a gouty arthritis model in rat by MSU injection, which has been widely used to apply in studies of acute gouty arthritis [16]. Male adult Sprague-Dawley rats were randomly allocated to three groups: (1) Control group, (2) Model group, (3) JQ-1 group. The dose of JQ-1 was decided based on our preliminary experiments and the published studies [17]. $1 \mathrm{~h}$ after pretreatment of JQ-1, animals were injected with $100 \mu \mathrm{l}$ MSU crystals (500 $\mu \mathrm{g} / \mathrm{ml}$ ) dissolved in sterile saline in ankle joint. After MSU stimulation, the circumference of ankle joint was measured at $0,2,4,8,12,24 \mathrm{~h}$. Mechanical hyperalgesia assay was performed by Von Fray assay [18]. After animals were sacrificed, synovium samples were collected for following analysis.

\section{Hematoxylin-eosin (H\&E) staining}

The synovium samples were fixed in the $4 \%$ paraformaldehyde solution immediately, and then embedded with paraffin. Subsequently, $5 \mu \mathrm{m}$ paraffin sections were dewaxed, rehydrated, and stained with $\mathrm{H} \& \mathrm{E}$ according to the protocol as our previous study [19]. After that, the histopathological evaluation was carried out.

\section{Cell Culture}

THP-1 cell line (human myeloid leukemia mononuclear cells) purchased from American Type Culture Collection (ATCC, USA), were maintained at $37^{\circ} \mathrm{C}$ under an atmosphere of $5 \% \mathrm{CO}_{2}$ in RPMI1640 medium containing 10\% fetal bovine serum (Gibco), $100 \mathrm{IU} / \mathrm{ml}$ penicillin, and $100 \mathrm{IU} / \mathrm{ml}$ streptomycin. THP-1 cells were performed followed by pre-differentiation for $48 \mathrm{~h}$ in supplemented RPMI 1640 containing $100 \mathrm{ng} / \mathrm{ml}$ phorbol 12-myristate 13-acetate (PMA) (Sigma-Aldrich). Then, the matured THP-1 cells were incubated under JQ-1 $(1 \mu \mathrm{M})$ for $1 \mathrm{~h}$, followed by the stimulation of MSU $(500 \mu \mathrm{g} / \mathrm{ml})$ for 12 hours prior to the following analysis.

\section{Transfection}

BRD4 siRNA (Genepharma, Shanghai) was used to transfect THP-1 cells at a confluency of $70 \%-90 \%$ 
using Lipofectamine 2000 (Life Technologies), according the manufacturer's instructions. After MSU stimulation for $12 \mathrm{~h}$, cells were collected for the future analysis.

\section{IL-1 $\beta$ assay}

The levels of IL-1 $\beta$ in rat synovial tissue homogenate and THP-1 cell supernatant were examined with enzyme-linked immunosorbent assay kit (R\&D, USA) according to the manufacturer's instructions.

\section{Pyroptosis assay}

Active Caspase- 1 and PI fluorescence in vivo and in vitro are necessary for pyroptosis characterization. We measured the active caspase- 1 level in cell suspensions with Caspase-1 Detection Kit (ImmunoChemistry Technologies, USA) using flow cytometry according to the manufacturer's instructions. And membrane pores induced by pyroptosis were marked by propidium iodide staining (BD, USA).

\section{Western blot}

The synovium samples and THP-1 cells collected from each group were taken to western blotting experiment. Briefly, samples were lysed in a RIPA buffer and the supernatant was collected after centrifugation. The extracted protein was separated by sodium dodecyl sulphate-polyacrylamide gel electrophoresis (SDS-PAGE) and transferred to polyvinylidene difluoride (PVDF) membranes (Millipore, USA), followed by membrane blocking with 5\% milk dissolved in Tris Buffered saline Tween (TBST) solution for $2 \mathrm{~h}$ at room temperature. Primary antibodies incubation was performed at $4{ }^{\circ} \mathrm{C}$ overnight, as listed in Table 1. Membranes received a $2 \mathrm{~h}$ incubation with horseradish peroxidaseconjugated secondary antibodies, and then bands detection was showed with Image Lab software (Bio-Rad, USA).

Table 1. Antibodies used for Western blot analysis

\begin{tabular}{lll}
\hline Company & Description & Catalog number \\
\hline BIOSS Biotech & Rabbit NLRP3 antibody & bs-6655R \\
(Beijing, P. R. China) & Rabbit ASC antibody & bs-6741R \\
& Rabbit caspase-1 antibody & bs-6368R \\
& Rabbit BRD4 antibody & bs-9759R \\
& Rabbit IL-1 $\beta$ antibody & bs-6319R \\
& Rabbit GSDMD antibody & bs-14287R \\
Cell Signaling Technology & Rabbit p65 NF-kB antibody & $\# 8242$ \\
(Boston, MA, USA) & Rabbit p-p65 NF-kB antibody & \#3033 \\
Bioword Technology, Inc & Rabbit $\beta$-actin antibody & AP0060 \\
(Louis Park, MN. USA) & Goat anti-rabbit IgG HRP & BS13278 \\
\hline
\end{tabular}

\section{Immunofluorescence}

The collected synovial tissues were fixed, embedded and sectioned. After MSU exposure, the THP-1 cells were fixed with $4 \%$ paraformaldehyde solution for 20-30 min. Subsequently, permeabilization was carried out with Triton X-100 for $30 \mathrm{~min}$, followed by incubation with blocking solution and primary antibodies at a 1: 50 dilution at 4 ${ }^{\circ} \mathrm{C}$ overnight. Tissue as well as cell samples were then incubated with secondary antibodies conjugated with Alexa Fluor dye at a 1:200 dilution, as listed in Table 2. After DAPI staining, fluorescent images were visualized usinga confocal laser scanning microscope (LSM 700, Zeiss, CA, USA).

Table 2. Antibodies used forimmunofluorescence

\begin{tabular}{|c|c|c|}
\hline Company & Description & $\begin{array}{l}\text { Catalog } \\
\text { number }\end{array}$ \\
\hline $\begin{array}{l}\text { Santa Cruz Biotechnology, Inc } \\
\text { (Dallas, TX, USA) }\end{array}$ & Goat NLRP3 antibody & sc-34410 \\
\hline \multirow[t]{3}{*}{ AbcamInc (Cambridge, UK) } & $\begin{array}{l}\text { RabbitASC antibody } \\
\text { Donkey Anti-Goat IgG H\&L } \\
\text { (Ex:495nm, Em:519nm) }\end{array}$ & $\begin{array}{l}\text { sc-22514-R } \\
\text { Ab150129 }\end{array}$ \\
\hline & $\begin{array}{l}\text { Donkey Anti-Rabbit IgG H\&L } \\
\text { (Ex:652nm, Em:668nm) }\end{array}$ & Ab150075 \\
\hline & $\begin{array}{l}\text { Goat Anti-Mouse IgG H\&L } \\
\text { (Ex:578nm, Em:603nm) }\end{array}$ & Ab175473 \\
\hline
\end{tabular}

\section{Statistical analysis}

Data have been presented as mean values \pm standard deviation. One-way analysis of variance (ANOVA) with Tukey multiple comparison test is used for data analysis, with $p$ value $<0.05$ considered to be significant.

\section{Results}

\section{Pyroptosis processing shifts under BRD4 inhibition in vitro}

It is firstly reported that BRD4 inhibition could contribute to the acute gouty arthritis therapy with the regulation of pyroptosis processing. As a novel programmed cell death, pyroptosis has been recognized to be dependent on Caspase- 1 activation and facilitate membrane disruption with positivity for propidium iodide (PI) staining [19]. First of all, we conducted the pyroptosis assay in the MSU-induced THP-1 cells to assess if BRD4 inhibitor JQ-1 could suppress the onset of pyroptosis. Pyroptotic cells exhibited PI and Caspase- 1 double positivity in flow cytometry detection [20]. FLICA 660-YVAD-FMK was used to quantify active caspase- 1 and PI dye was used to mark cells with membrane pores. MSU exposure induced a significant elevation in the amount of double positive cells in comparison with normal ones (Figure 1A-1C). And aforementioned alterations were reversed by the addition of JQ-1, meanwhile, the negative correlation with Caspase- 1 activation could also be verified by the reduction of IL- $1 \beta$ secretion under BRD4 suppression, suggesting the effect of BRD4 inhibition works against MSU-induced pyroptotic cell death (Figure 1D). 
A

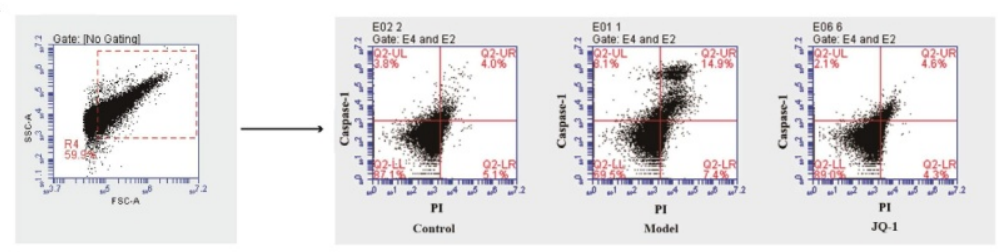

B

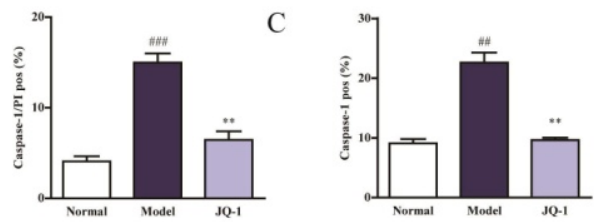

$\mathrm{D}$

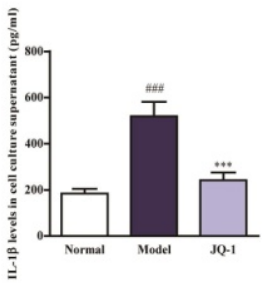

E

Normal Model JQ-1
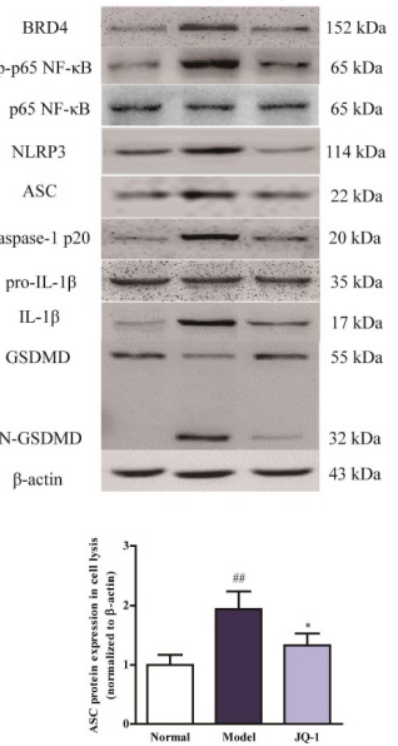
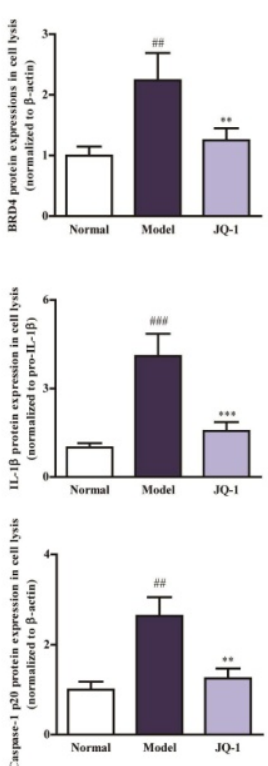
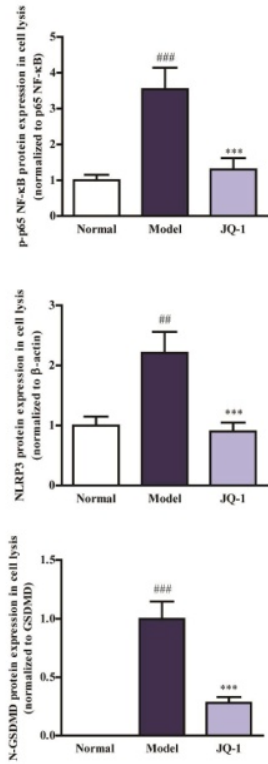

MERGED

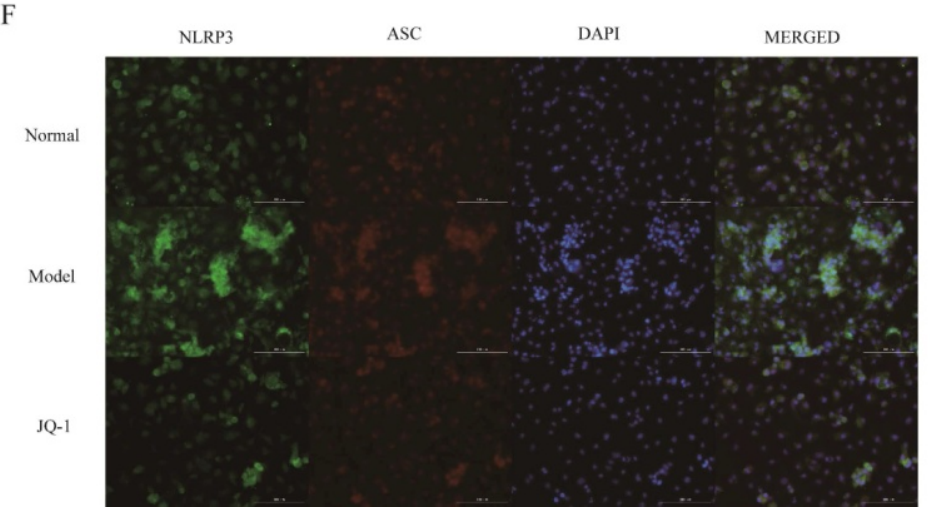

Figure 1. Pyroptosis processing shifts during BRD4 inhibition in MSU-induced THP-1 Human Promonocytes. After PMA treatment, the THP-1 cells were incubated with the absence or presence of serum free medium containing JQ-1 $(1 \mu \mathrm{M})$ for $1 \mathrm{~h}$, followed by the stimulation with MSU (500 $\mu \mathrm{g} / \mathrm{ml})$ for $12 \mathrm{~h}$. The rate of pyroptotic cell death was examined with PI and active Caspase-1 double staining by flow cytometry (A). Positive Caspase-1/PI fluorescence intensity of samples in THP-1 cells (B). Positive Caspase-1 fluorescence intensity of samples in THP-1 cells (C). The culture supernatants level of IL-1 $\beta$ was detected using the ELISA kit (D). The expression of BRD4 and NLRP3 inflammasome activation was measured by western blotting $(\mathrm{E})$. The relative optical densities of specific proteins were recorded. Representative confocal microscopy photographs of THP-1 cells with immunofluorescence changes are presented (F). NLRP3 protein was marked with the donkey anti-goat polyclonal secondary antibody conjugated with Alexa Fluor 488 (Green). ASC protein was marked with the donkey anti-rabbit polyclonal secondary antibody conjugated with Alexa Fluor ${ }^{\circledR} 647$ (Red). The data was presented as means $\pm S D$ s. Compared with normal group: $\# P<0.05,{ }^{*} P<0.01, \ldots+P<0.001$. Compared with Model group: ${ }^{*} P<0.05,{ }^{* *} P<0.01$, ${ }^{* * *} P<0.001$. Each group $(n=4)$. 


\section{NLRP3 inflammasome activation occurs during MSU-induced pyroptosis}

It has been demonstrated by emerging evidence that a positive correlation exists between the progression of gouty arthritis and NLRP3 inflammasome activation [21]. Considering the up-stream role of NLRP3 inflammasome activation for caspase- 1 eruption, corresponding analyses were performed to further investigate whether NLRP3 inflammasome was involved in the JQ-1 resistance to MSU-challenged pyroptosis in vitro [6]. MSU caused the up-regulation of BRD4, phosphorylation of p65 $\mathrm{NF}-\mathrm{kB}$, induction of NLRP3 inflammasome followed by cleavage of GSDMD in THP-1 cells compared with normal group, which were obviously ameliorated by JQ-1 intervention, indicating that JQ-1 could influence the inflammatory response by mediating the upstream NLRP3 inflammasome inactivation (Figure 1E). As shown in Figure 1F, immunofluorescence data also confirmed JQ-1 blocked the assembly of NLRP3 inflammasome characterized by less co-localization of NLRP3 and ASC. Moreover, lower fluorescence intensity of NLRP3, ASC exhibited after JQ-1 pretreatment in MSU-induced THP-1 cells, implicating that blocking BRD4 expression could prevent the Caspase-1-dependent pyroptosis progression via inhibiting NLRP3 inflammasome activation.

\section{The effect of JQ-1 on acute gouty arthritis in vivo}

After pretreatment with JQ-1, joint circumference detection was performed at $0,2,4,8$, $12,24 \mathrm{~h}$ after MSU crystals injection to evaluate the severity of synovial pathology. As revealed in Figure 2A-2C, joint circumference in model group significantly increased at 2, 4, $8 \mathrm{~h}$ and enlarged to peak value at $12 \mathrm{~h}$ point than those of normal group, indicating the enhanced joint swelling in response to MSU stimulation. On the contrary, BRD4 inhibitor restored the joint circumference to normal level with merely 1.05-fold than those of normal group. In comparison with normal ones, mechanical withdrawal of model rats declined rapidly at 2, 4, $8 \mathrm{~h}$ time points and decreased to $0.52 \mathrm{~g}$ at $12 \mathrm{~h}$ after MSU injection. This MSU-induced tendency of pain reaction was apparently reversed to some extent by administration of JQ-1 at different time points. Meanwhile, it was also confirmed by histopathological observation, evidenced by the suppressed synovial hyperplasia and severe neutrophil infiltration of JQ-1 samples (Figure 2E). Additionally, an improvement of MSU-induced elevation of IL-1 $\beta$ concentration was found in JQ-1 rats, affirming the protection of JQ-1 from gouty arthritis (Figure 2D).
BRD4 inhibitor prevents the pyroptosis death accompanied with suppressed NLRP3 inflammasome activation in vivo

Considering the connection between IL-1 $\beta$ and Caspase-1, flow cytometry was used to investigate whether BRD4 inhibitor have an impact on Caspase-1-dependent pyroptosis in animal model of gouty arthritis (Figure 3A). Macrophages were classified based on positive staining for CD68. Interestingly, the quantity pyroptotic macrophages (double-positive) was remarkably elevated in synovial tissue of rats injected by MSU, which was pulled down closer to normal level by the intervention of JQ-1 (Figure 3B, 3C). And this inhibition of JQ-1 injection in the Caspse-1 activation in synovium lysates of model animals was confirmed using western blotting and immunofluorescence. Hence, we proposed that Brd4 expression might be involved in relative mechanism of pyroptosis.

Pyroptosis has not been characterized in acute gouty arthritis pathologies, nor has its relevance to MSU-challenged in vivo model been elucidated. And it has been reported that NLRP3 inflammasome could induce this inflammatory cascade-related pyroptosis [22]. Consistent with cell experiments, immunoblotting analysis revealed an apparent up-regulation in Brd4 expression, p65 NF-kB phosphorylation, NLRP3 inflammasome activation and cleavage of GSDMD under MSU exposure in synovium specimens. Conversely, the blockage of Brd4 by JQ-1 down-regulated these expressions in synovium, suggesting that the effect of JQ-1 on pyroptosis might be mediated by NLRP3 inflammasome signaling (Figure 4A). Besides, Figure $4 \mathrm{~B}$ showed the JQ-1 treatment could block activated NLRP3 signaling after MSU stimulation, as evidenced by reduced fluorescence intensity of NLRP3, ASC and their co-localization in synovium (Figure 4B). The data above indicated that the formation of NLRP3 inflammasome complex was blocked in the presence of BRD4 inhibitor JQ-1, suggesting the role of BRD4 in pyroptosis was related to the NLRP3 signaling cascade.

\section{BRD4 knockdown is resistant to MSU stimulation in vitro}

To address the role of BRD4 in acute gouty arthritis, we examined pyroptotic response in BRD4 knockdown (KD) THP-1 cells. The stability of Caspase-1 activation and membrane integrity was observed in BRD4 knockdown THP-1 cells once MSU stimulated (Figure 5A-5C). In contrast, it was without BRD4 transfection that MSU stimulation could still augment pyroptosis reaction with the more potent positivity of Caspase-1 and PI staining, suggesting 
that the role of BRD4 in governing the regulation of pyroptosis onset. By the way, as shown in the Figure $5 \mathrm{D}$, the secretion of IL- $1 \beta$ in cell supernatant was also unchanged in the presence of BRD4 KD cells under MSU exposure, which was consist with our hypothesis.

And we found the western blotting and immunofluorescence data uncovered the similar phenomenon in which BRD4 knockdown abolished relevant alterations of the p65 NF-kB phosphorylation, NLRP3 inflammasome activation and cleavage of GSDMD, which were brought by MSU administration, demonstrating that BRD4 could regulate the p65 NF- $\mathrm{kB}$ phosphorylation and its downstream signaling cascade- NLRP3 inflammasome function (Figure 5E, 5F). Notably, NLRP3 could directly recognize MSU as a danger signal and subsequently induce inflammatory response, which might contribute to the slight activation of NLRP3 in
siBRD4+MSU group compare to siBRD4 alone.

\section{Discussion}

Our study is the first research to reveal the role of BRD4 in MSU-induced NLRP3 inflammasome activation in acute gouty arthritis. Above results section demonstrated that enhanced NLRP3 activation caused by MSU crystals would lead to ankle joints swelling, severe synovial inflammation with an apparent neutrophilic infiltration and intense pyroptotic death progression characterized by the increased positivity of PI and Caspase-1 double-staining. Meanwhile, these effects were predominantly reversed through the pretreatment with BRD4 inhibitor JQ-1, suggesting its involvement of Caspase-1-dependent pyroptotic cell death in mechanisms of acute gouty arthritis.

$\mathbf{A}$

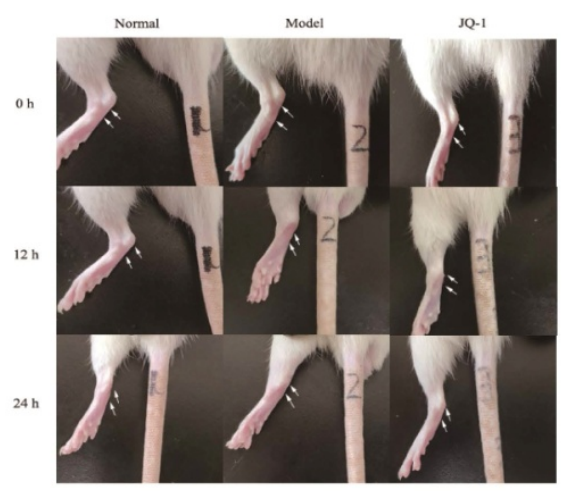

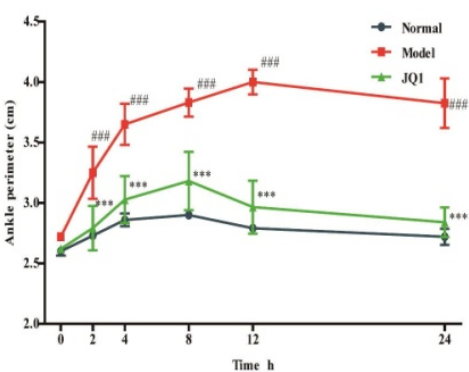

C

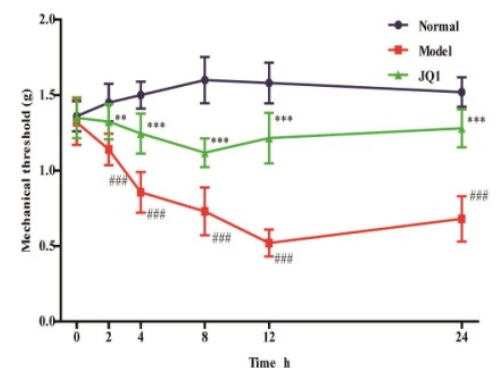

D

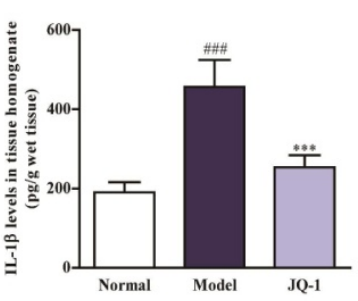

E

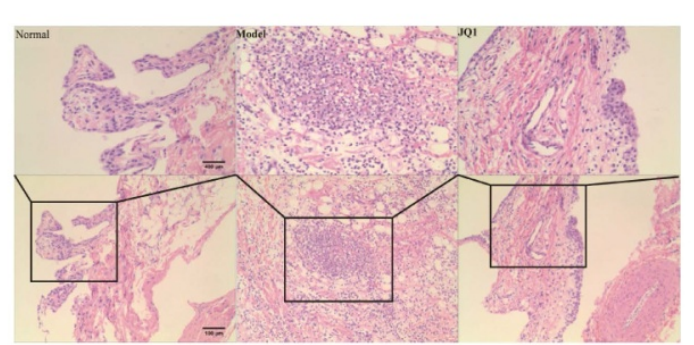

Figure 2. The effect of BRD4 inhibitor on MSU-induced acute gouty arthritis in vivo. $1 \mathrm{~h}$ after pretreatment of JQ-1, animals were injected with MSU crystals to induce acute gouty arthritis. Representative photographs to show the swelling of joints are presented (A). The injected ankle joint circumference of each rat was determined at $0,2,4,8,12$, $24 \mathrm{~h}$ after MSU stimulation (B). Mechanical hyperalgesia, observed as an increase in nociceptive response, was assessed by Von Fray assay at 0, 2, 4, 8, 12, 24 h after MSU stimulation (C). The IL-1 $\beta$ level in synovium homogenate was detected by the ELISA kit (D). Representative photographs of histopathologic changes in synovium are presented

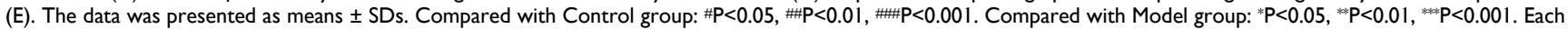
group $(n=6)$. 
A
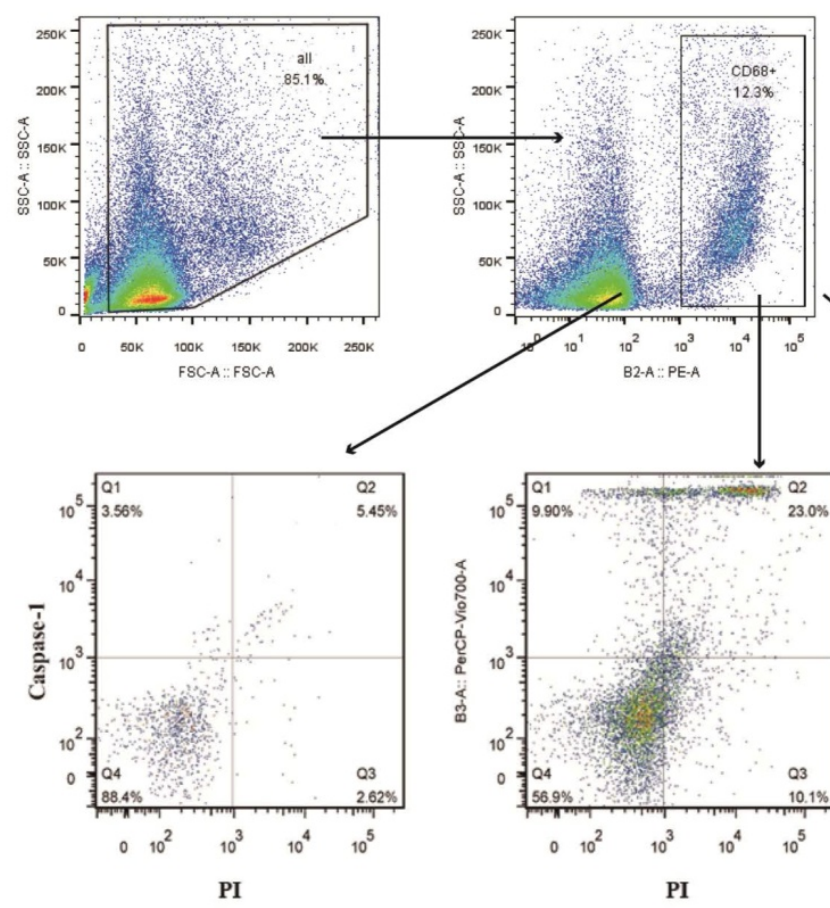

Normal

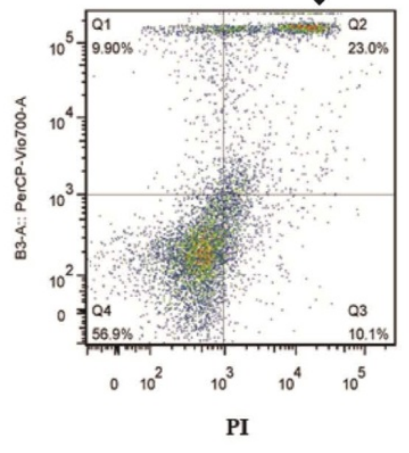

Model

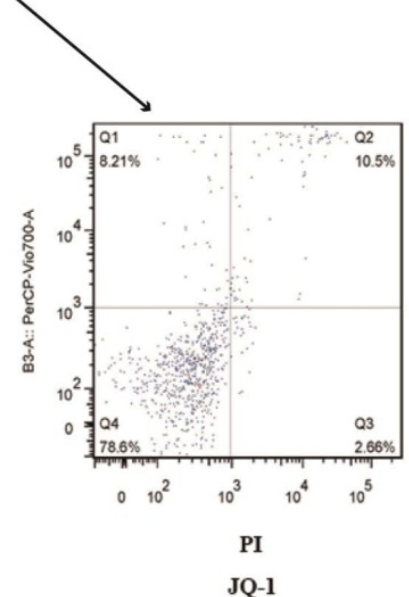

$\mathrm{B}$

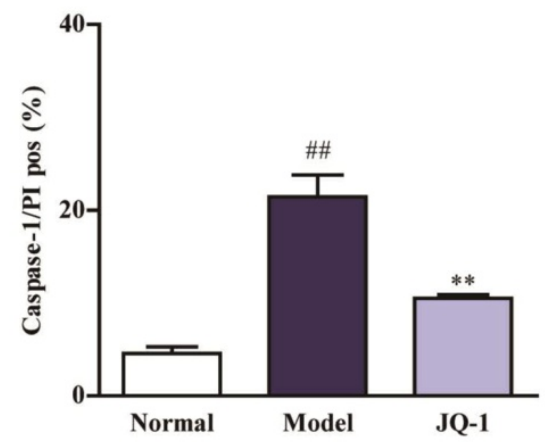

$\mathrm{C}$

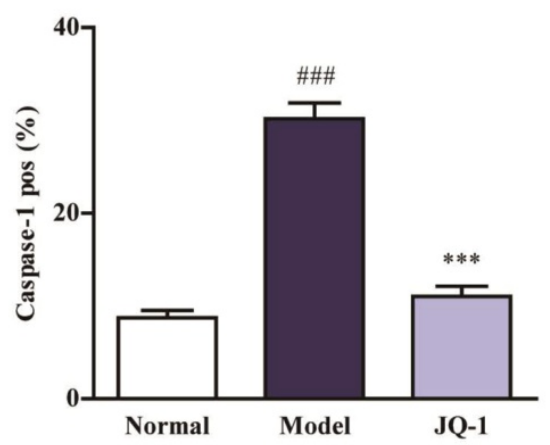

Figure 3. BRD4 inhibitor regulates the pyroptosis death in synovial tissue. $1 \mathrm{~h}$ after pretreatment of JQ-1, animals were injected with MSU crystals to induce acute gouty arthritis. To determine the rate of pyroptotic cell death in synovial tissue, macrophages were classified based on positive staining for CD68. Then pyroptosis assay was performed in the macrophages obtained from synovium using PI and active Caspase-1 double staining by flow cytometry (A). Positive Caspase-1/PI fluorescence intensity of samples (B). Positive Caspase-1 fluorescence intensity of samples (C). The data was presented as means \pm SDs. Compared with Control group: \#P<0.05, \#P<0.01, \#\#<0.001. Compared with Model group: ${ }^{*} P<0.05,{ }^{* *} P<0.01,{ }^{* * *} P<0.001$. Each group $(n=4)$.

MSU-induced pyroptosis, a kind of programmed cell death, is usually marked by activated caspase- 1 and membranolysis, thus, double staining detection of caspase-1+PI has been used to evaluate of NLRP3-related pyroptosis, which has been regarded as dependable method [20]. As a Capase-1-mediated programmed death, pyroptosis process is usually accompanied by pore formation of plasma membrane, followed by permeability alterations and subsequent cellular leakages. And PI can go through damaged plasma membrane and combine with DNA, but not complete membrane structure. Therefore, based on the selective caspase-1 detection, PI staining also contributed to evaluate the pyroptotic death. On the other hand, active Caspase- 1 performed the cleavage of the amino-terminal gasdermin-N from GSDMD, which was necessary for pyroptosis [11]. Therefore, we detected protein expression of GSDMD-N in order to determine whether cell would undergo pyroptosis. Once sensing according stimuli, after priming step, the relevant NLR (NOD-like receptors, NLRs) can oligomerize to assemble an inflammasome complex followed by the maturation of pro-Caspase-1 [6]. Subsequently, active Caspase- 1 serves to cleave the pro-inflammatory cytokines IL-1 $\beta$ into its bioactive forms. Meanwhile, active IL-1 $\beta$ release could also 
promote a potent inflammatory response in pyroptosis [23]. Currently, enhanced NLRP3 inflammasome could mediate caspase- 1 activation, interleukin-1 $\beta$ secretion, and pyroptosis by the addition of NLRP3 stimuli, which contributed to the pathology of rheumatoid arthritis in vivo [24]. And hepatocyte pyroptotic cell death has been reported as a novel mechanism of NLRP3 inflammasomemediated liver damage [20]. Our data suggest that NLRP3 inflammasome activation would also exaggerate the development of acute gouty arthritis, accompanied with pyroptosis eruption.
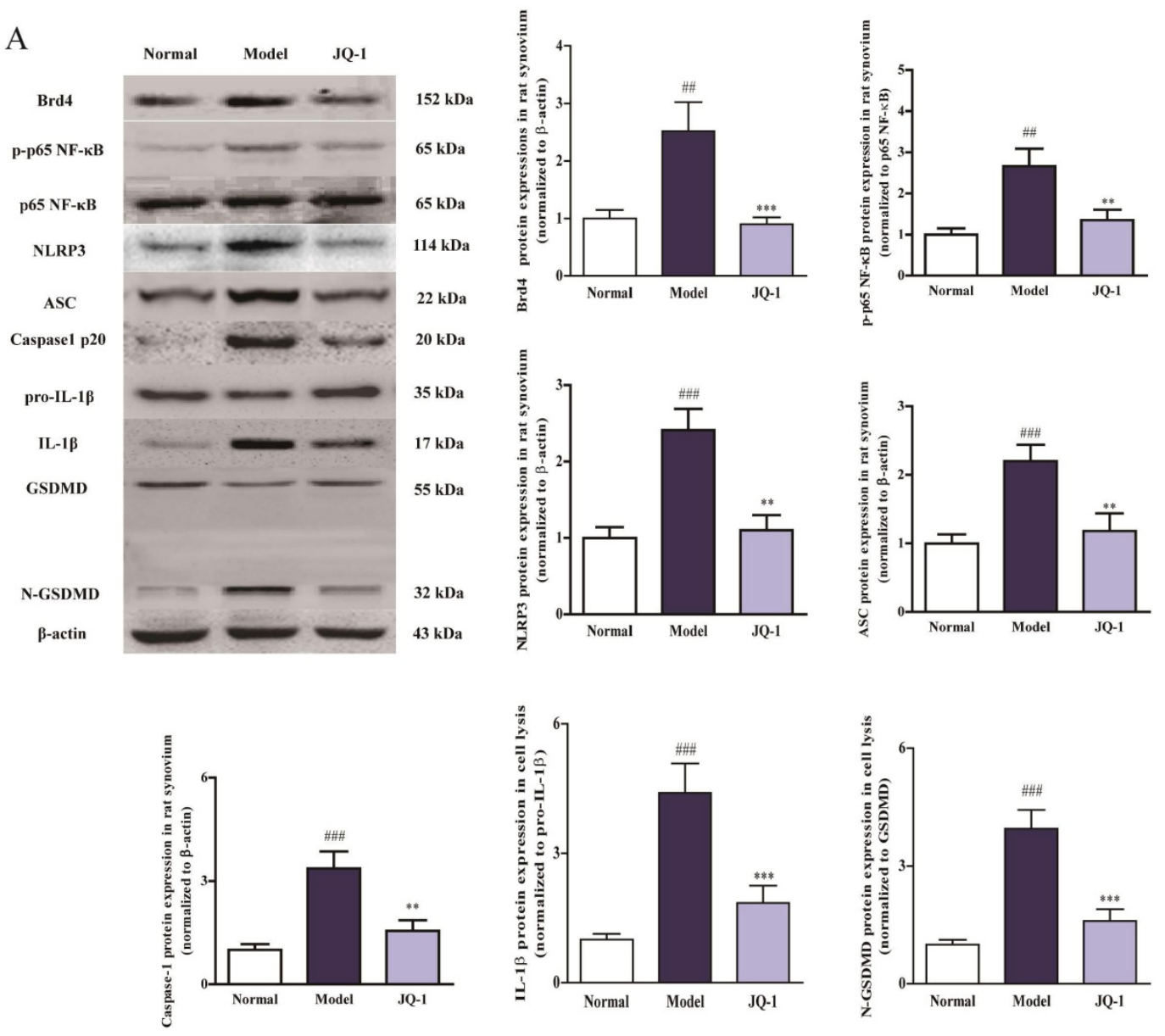

$\mathrm{B}$
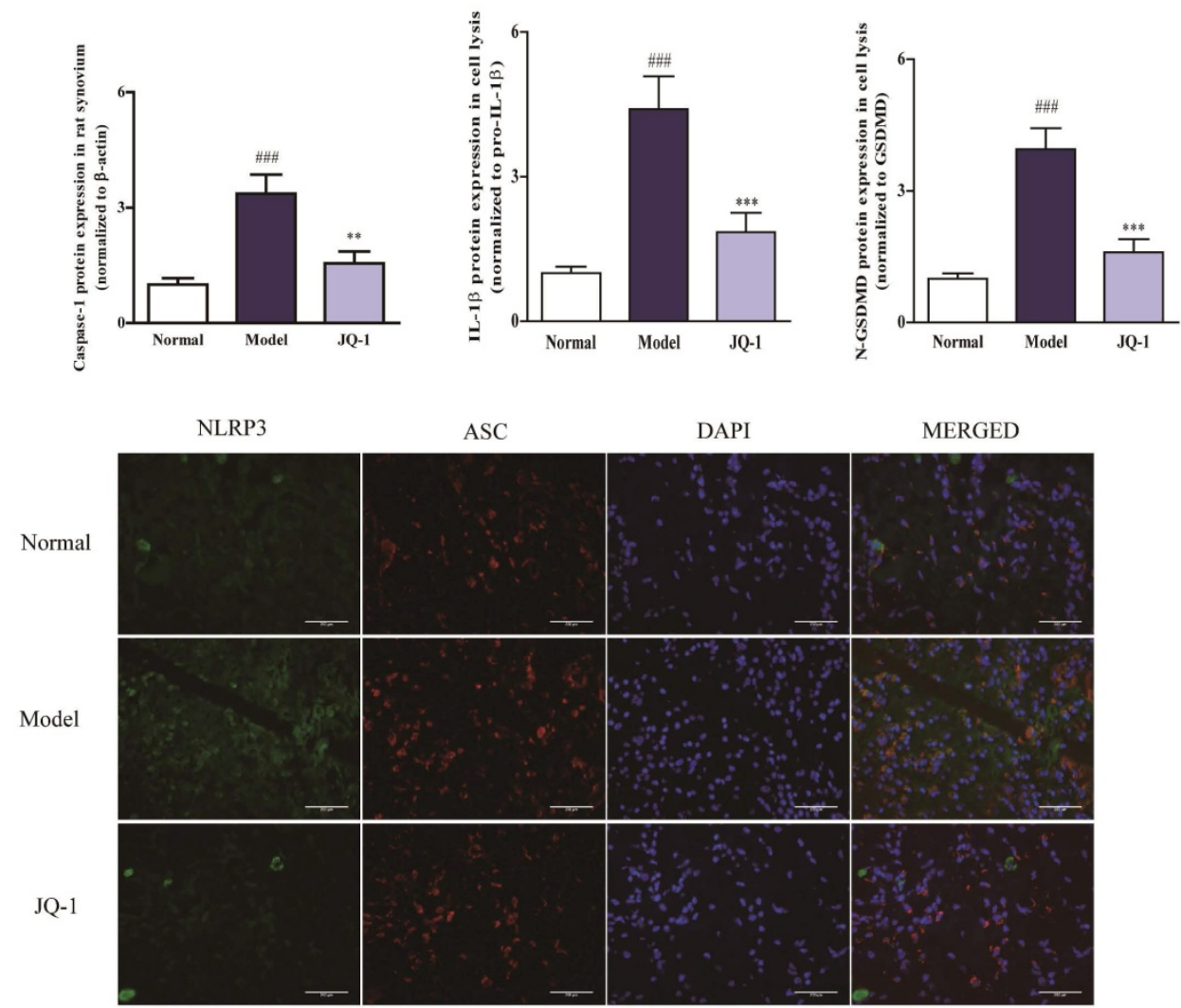

Figure 4. Suppressed NLRP3 inflammasome activation in acute gouty arthritis during Brd4 inhibition in acute gouty arthritis. $1 \mathrm{~h}$ after pretreatment of JQ-1, animals were injected with MSU crystals to induce acute gouty arthritis. The protein expression of Brd4 and NLRP3 inflammasome pathway in synovium after the JQ-1 treatment (A). The relative optical densities of specific proteins were recorded. NLPR3 inflammasome activation in synovial tissues was evaluated with immunofluorescence staining (B). NLRP3 protein was marked with the donkey anti-goat polyclonal secondary antibody conjugated with Alexa Fluor $\AA 488$ (Green). ASC protein was marked with the donkey anti-rabbit polyclonal secondary antibody conjugated with Alexa Fluor ${ }^{\circledR} 647$ (Red). The data was presented as means \pm SDs. Compared with Control group: \#P<0.05, \#P<0.01, \#\#P<0.001. Compared with Model group: * $\mathrm{P}<0.05,{ }^{*} \mathrm{P}<0.01,{ }^{* * *} \mathrm{P}<0.001$. Each group $(\mathrm{n}=4)$. 
A

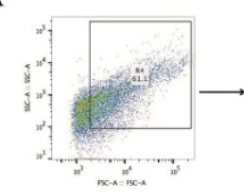

B

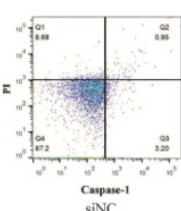

Caspase-
siNC

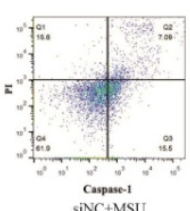

C

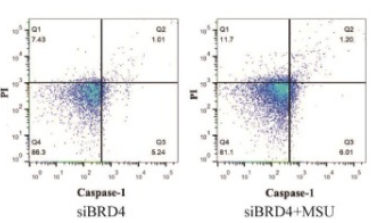

Caspase-1
siBRD4+MSU
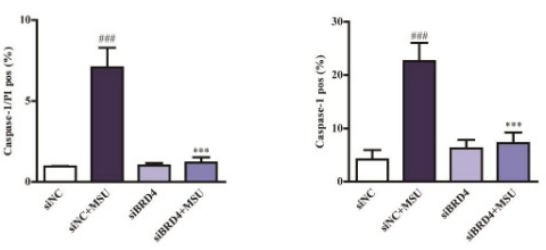

D

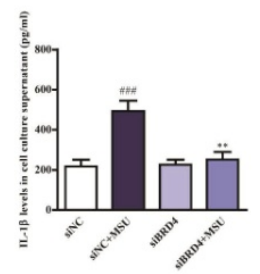

E
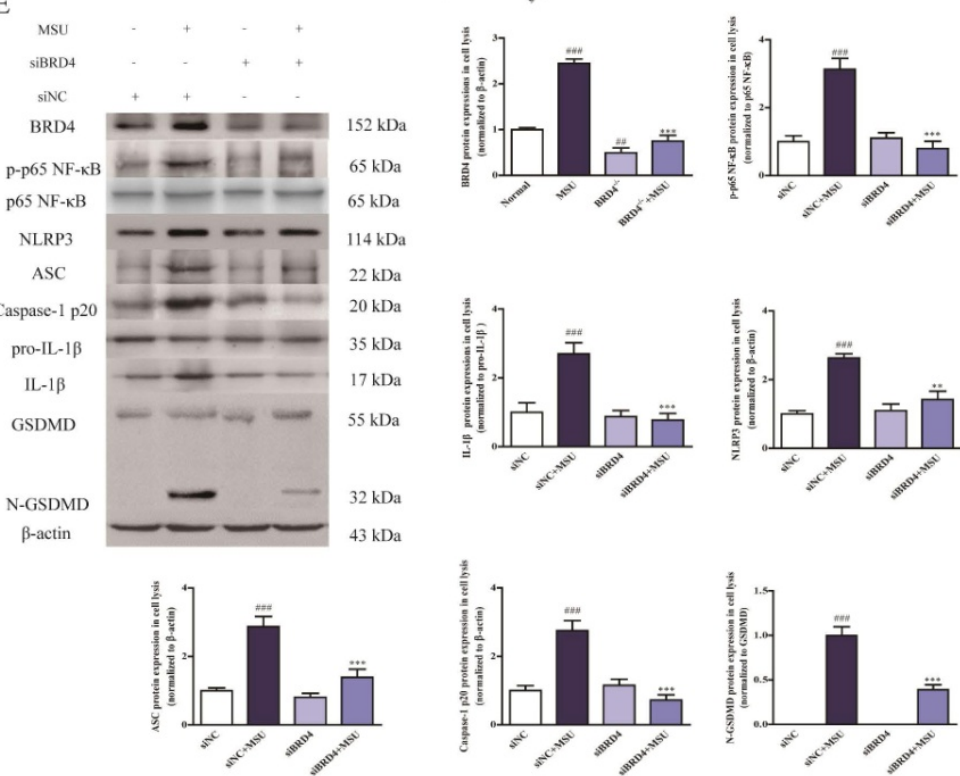

$152 \mathrm{kDa}$
$65 \mathrm{kDa}$

$65 \mathrm{kDa}$

$114 \mathrm{kDa}$

$22 \mathrm{kDa}$

$20 \mathrm{kDa}$

$35 \mathrm{kDa}$

$17 \mathrm{kDa}$

$55 \mathrm{kDa}$
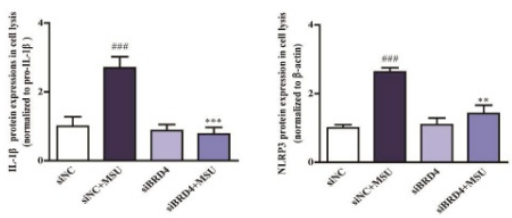

$43 \mathrm{kD}$
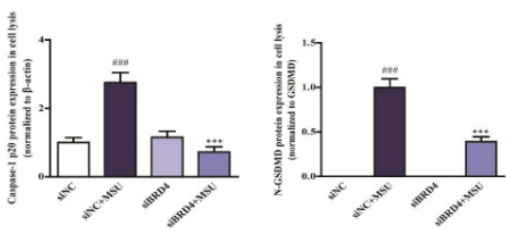

F

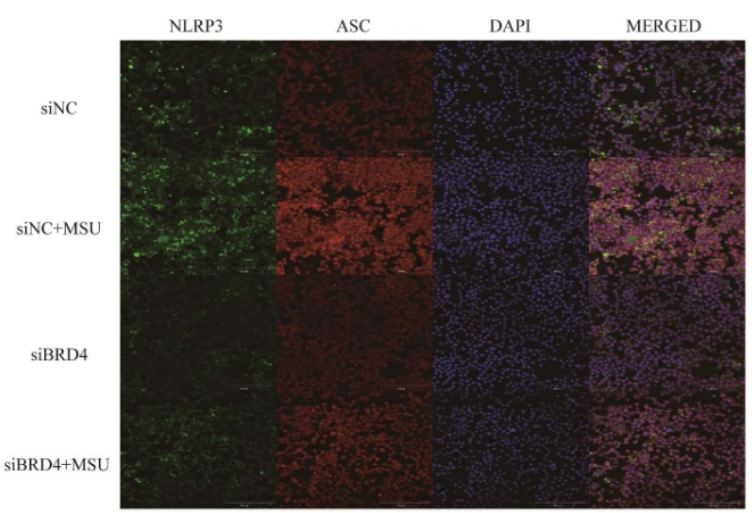

Figure 5. The effect of BRD4 knockdown on the pyroptosis assay. BRD4 siRNA was used to transfect THP-1 cells at a confluency of $70 \%-90 \%$ with Lipofectamine 2000 . After transfection for $24 \mathrm{~h}$, the THP-1 cells were stimulated by MSU $(500 \mu \mathrm{g} / \mathrm{ml})$ for $12 \mathrm{~h}$. The rate of pyroptotic cell death was examined with PI and active Caspase-1 double staining by flow cytometry (A). Positive Caspase-1/PI fluorescence intensity of samples in THP-1 cells (B). Positive Caspase-1 fluorescence intensity of samples in THP-1 cells (C). The culture supernatants level of IL-1 $\beta$ was detected using the ELISA kit (D). The expression of BRD4 and NLRP3 inflammasome activation was measured by western blotting (E). The relative optical densities of specific proteins were recorded. Representative confocal microscopy photographs of THP-1 cells with immunofluorescence changes are presented (F). NLRP3 protein was marked with the donkey anti-goat polyclonal secondary antibody conjugated with Alexa Fluor ${ }^{\circ} 488$ (Green). ASC protein was marked with the donkey anti-rabbit polyclonal secondary antibody conjugated with Alexa Fluor ${ }^{\circledR} 647$ (Red). The data was presented as means \pm SDs. Compared with Control group: \#P<0.05, \#P<0.01, \#\#<0.001. Compared with Model group: ${ }^{*} P<0.05$, * ${ }^{*} P<0.01,{ }^{* * *} P<0.001$. Each group $(n=4)$. 


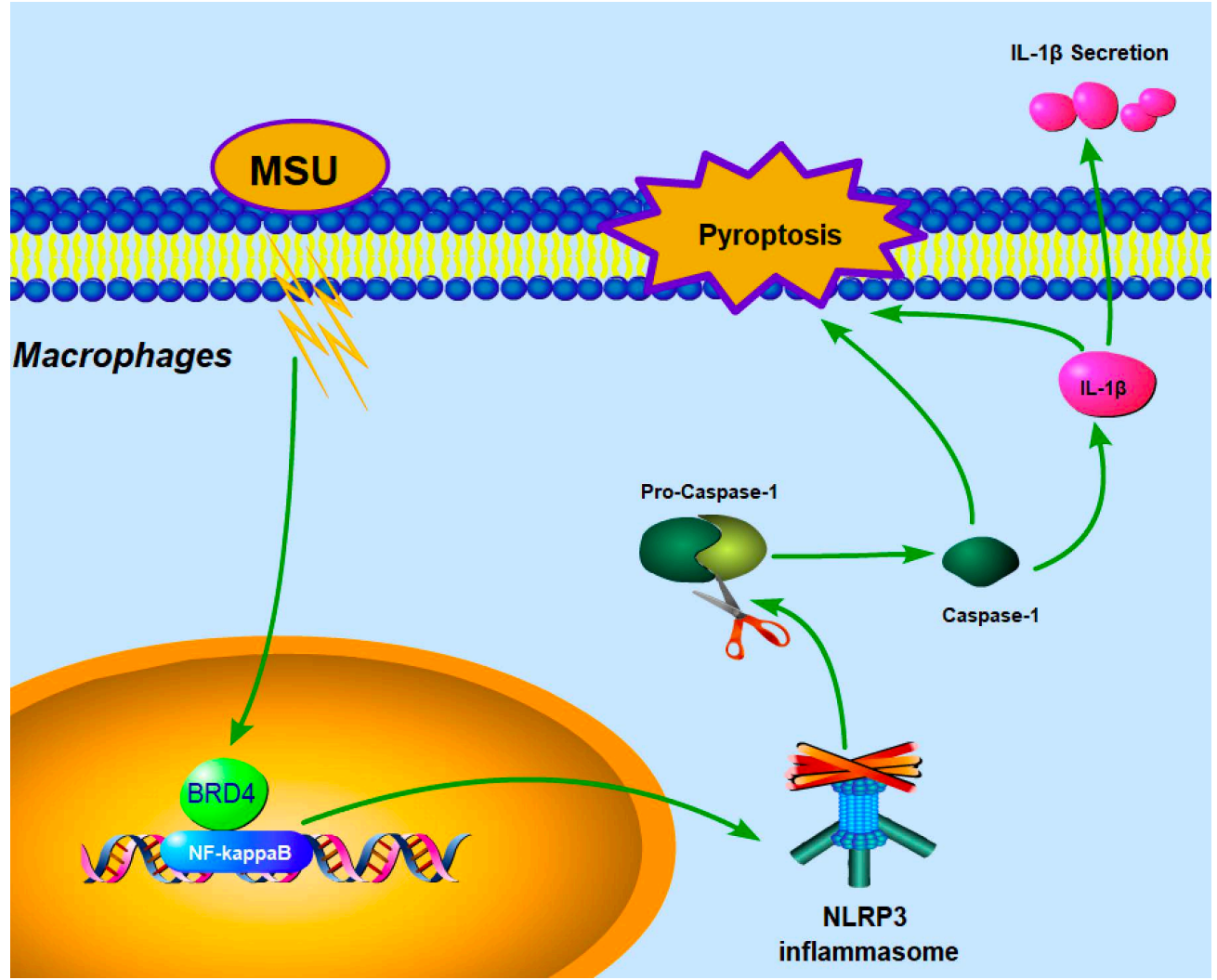

Figure 6. Proposed mechanism: BRD4 regulates NLRP3 inflammasome activation and IL-1 $\beta$ secretion during MSU stimulation.

In general, NLRP3 protein must be primed in prior to NLRP3 inflammasome activation [6]. Priming is required for NLRP3 inflammasome to increase the cellular NLRP3 level in response to stimulus, like, LPS binding to TLR4 [25]. Previous studies have reported the critical role of NF-kB in synovial inflammation [26, 27]. NF-KB has been well known to serve as a mediator of inflammation and energy failure, which governs the production of pro-inflammatory cytokine [28]. As the inhibitor of NF- $\mathrm{kB}, \mathrm{I \kappa Ba}$ is dominated by IKB kinase (IKK) complex consisting of IKK- $\alpha$ and IKK- $\beta$. Upon stimulation, the NF-KB subunit p65 would be activated by the phosphorylation and degradation of the IKBa [29]. In general, NF- $\mathrm{KB}$ is distributed in the cytosol binding to its inhibitor ІкB. $\mathrm{NF}-\mathrm{kB}$ separates from IкBa under inflammation, followed by the translocation into nucleus to regulate the transcription of pro-inflammatory cytokines, which will promote the activation of NLRP3 inflammasome signaling. In the current study, results showed the effect of BRD4 knockdown on the phosphorylation of NF-kB with unchanged IL-1 $\beta$ release in vivo and in vitro, confirming the relevance of BRD4 expression to NF-KB activation. Additionally, downstream NLRP3 inflammasome activation turned out to be invalid once BRD4 was pulled down, implying that NF-kB phosphorylation followed by NLRP3 inflammasome activation are crucial factor accounting for the protective effect of BRD4 inhibition under MSU stimulation.

Our current data demonstrated that BRD4 suppression could partially counteract the severity of pyroptotic death in acute gouty arthritis via the BRD4/NF-KB/NLRP3/GSDMD axis. Our proposed hypothesis about mechanisms was exhibited as shown in the Fig 6. Similarly, recent studies indicated the marked potency of BRD4 inhibitor JQ-1 against synovial inflammation by blocking IKB kinase-mediated NF-KB translocation in rheumatoid fibroblast-like synoviocytes $[14,15]$. Notably, affecting the acetylated histones recognition of the BET family proteins would attribute to regulate pro-inflammatory gene expression [30]. And JQ1 was also found to prevent the "cytokine storm" in endotoxemic mice to rescue animals from LPS-induced inflammation, suggesting that targeting BET proteins with the small-molecule inhibitor could improve inflammatory conditions [31].

In conclusion, articular Brd4 expression was correlated with the joint lesion and pyroptosis cascade events in MSU-induced acute gouty arthritis. The protective effect of BRD4 inhibition by JQ-1 or siRNA on MSU-induced model might be mediated via the BRD4/NF-kB/NLRP3/GSDMD axis. 


\section{Supplementary Material}

Supplementary figure.

http://www.ijbs.com/v16p3163s1.pdf

\section{Acknowledgements}

This research was supported by Natural Science Foundation of China (Grants 81773745, 81773826), The Drug Innovation Major Project (Grants 2018ZX 09711001), 111 Incubation Project (BC2018024) and "Double First-Class" University project (CPU2018GF02).

\section{Competing Interests}

The authors have declared that no competing interest exists.

\section{References}

1. Choi HK, Mount DB, Reginato AM. Pathogenesis of gout. Annals of Internal Medicine. 2005; 143: 499-516.

2. Martillo MA, Nazzal L, Crittenden DB. The crystallization of monosodium urate. Curr Rheumatol Rep. 2014; 16: 400.

3. Wu XH, Wang CZ, Wang SQ, et al. Anti-hyperuricemia effects of allopurinol are improved by Smilax riparia, a traditional Chinese herbal medicine. J Ethnopharmacol. 2015; 162: 362-8.

4. Liu Y, Tang H, Liu X, et al. Frontline Science: Reprogramming COX-2, 5-LOX, and CYP4A-mediated arachidonic acid metabolism in macrophages by salidroside alleviates gouty arthritis. Journal of leukocyte biology. 2019; 105: $11-24$

5. Marchetti C, Swartzwelter B, Koenders MI, et al. NLRP3 inflammasome inhibitor OLT1177 suppresses joint inflammation in murine models of acute arthritis. Arthritis Res Ther. 2018; 20: 169.

6. Guo H, Callaway JB, Ting JP. Inflammasomes: mechanism of action, role in disease, and therapeutics. Nat Med. 2015; 21: 677-87.

7. Strowig T, Henao-Mejia J, Elinav E, et al. Inflammasomes in health and disease. Nature. 2012; 481: 278-86

8. Szabo G, Csak T. Inflammasomes in liver diseases. J Hepatol. 2012; 57: 642-54.

9. Cookson BT, Brennan MA. Pro-inflammatory programmed cell death. Trends Microbiol. 2001; 9: 113-4.

10. Fink SL, Cookson BT. Apoptosis, pyroptosis, and necrosis: mechanistic description of dead and dying eukaryotic cells. Infect Immun. 2005; 73: $1907-16$

11. Shi J, Zhao Y, Wang K, et al. Cleavage of GSDMD by inflammatory caspases determines pyroptotic cell death. Nature. 2015; 526: 660-5.

12. Fink SL, Cookson BT. Caspase-1-dependent pore formation during pyroptosis leads to osmotic lysis of infected host macrophages. Cell Microbiol. 2006; 8: 1812-25.

13. Hajmirza A, Emadali A, Gauthier A, et al. BET Family Protein BRD4: An Emerging Actor in NFkappaB Signaling in Inflammation and Cancer. Biomedicines. 2018; 6 .

14. Zhang QG, Qian J, Zhu YC. Targeting bromodomain-containing protein 4 (BRD4) benefits rheumatoid arthritis. Immunology letters. 2015; 166: 103-8.

15. Xiao $Y$, Liang L, Huang M, et al. Bromodomain and extra-terminal domain bromodomain inhibition prevents synovial inflammation via blocking IkappaB kinase-dependent NF-kappaB activation in rheumatoid fibroblast-like synoviocytes. Rheumatology (Oxford). 2016; 55: 173-84.

16. Zhou M, Li S, Song $\mathrm{L}$, et al. 4-(2-(4-chlorophenyl)-1-((4-chlorophenyl)amino)ethyl)benzene-1, 3-diol is a potential agent for gout therapy as a dual inhibitor of XOD and NLRP3. Phytomedicine. 2018; 42 .

17. Jiang $\mathrm{Y}$, Zhu L, Zhang T, et al. BRD4 has dual effects on the HMGB1 and NF-KB signalling pathways and is a potential therapeutic target for osteoarthritis. Biochimica et Biophysica Acta (BBA) - Molecular Basis of Disease. 2017; 1863: 3001

18. Hoffmeister C, Silva MA, Rossato MF, et al. Participation of the TRPV1 receptor in the development of acute gout attacks. Rheumatology (Oxford). 2014; 53: 240-9.

19. Ine J, Miao EA. Pyroptotic cell death defends against intracellular pathogens. Immunological Reviews. 2015; 265: 130-42.

20. Wree A, Eguchi A, Mcgeough MD, et al. NLRP3 inflammasome activation results in hepatocyte pyroptosis, liver inflammation, and fibrosis in mice. Hepatology. 2014; 59: 898.

21. He $\mathrm{H}$, Jiang $\mathrm{H}$, Chen $\mathrm{Y}$, et al. Oridonin is a covalent NLRP3 inhibitor with strong anti-inflammasome activity. Nature Communications. 2018; 9.

22. Yin $\mathrm{L}, \mathrm{Bao} \mathrm{F}, \mathrm{Wu} \mathrm{J}$, et al. NLRP3 inflammasome-dependent pyroptosis is proposed to be involved in the mechanism of age-dependent isoflurane-induced cognitive impairment. Journal of neuroinflammation. 2018; 15: 266

23. Wen H, Miao EA, Ting JPY. Mechanisms of NOD-like Receptor-Associated Inflammasome Activation. Immunity. 2013; 39: 432-41.

24. Walle LV, Opdenbosch NV, Jacques P, et al. Negative regulation of the NLRP3 inflammasome by A20 protects against arthritis. Nature. 2014; 512: 69-73.

25. Bauernfeind FG, Horvath G, Stutz $A$, et al. Cutting edge: NF-kappaB activating pattern recognition and cytokine receptors license NLRP3 inflammasome activation by regulating NLRP3 expression. Journal of immunology (Baltimore, Md : 1950). 2009; 183: 787-91.

26. $\mathrm{Li} \mathrm{N}, \mathrm{Xu} \mathrm{O}$, Liu $\mathrm{O}$, et al. Leonurine attenuates fibroblast-like synoviocyte-mediated synovial inflammation and joint destruction in rheumatoid arthritis. Rheumatology. 2017; 56: 1417.

27. Han $Q$, Bing W, Di $Y$, et al. Kinsenoside screening with a microfluidic chip attenuates gouty arthritis through inactivating NF-KB signaling in macrophages and protecting endothelial cells. Cell Death \& Disease. 2016; 7: e2350.

28. Saito $\mathrm{T}$, Tanaka S. Molecular mechanisms underlying osteoarthritis development: Notch and NF-kappaB. Arthritis Res Ther. 2017; 19: 94.

29. Hinz $\mathrm{M}$, Scheidereit $\mathrm{C}$. The $\mathrm{I} \kappa \mathrm{B}$ kinase complex in NF- $\mathrm{KB}$ regulation and beyond. Embo Reports. 2014; 15: 46-61.

30. Nicodeme E, Jeffrey KL, Schaefer U, et al. Suppression of inflammation by a synthetic histone mimic. Nature. 2010; 468: 1119-23.

31. Belkina AC, Nikolajczyk BS, Denis GV. BET protein function is required for inflammation: Brd2 genetic disruption and BET inhibitor JQ1 impair mouse macrophage inflammatory responses. Journal of immunology (Baltimore, $\mathrm{Md}$ : 1950). 2013; 190: 3670-8. 